\title{
Protein structural dynamics by Magic-Angle Spinning NMR
}

\author{
Marta Bonaccorsi, Tanguy Le Marchand and Guido Pintacuda \\ Université de Lyon, Centre de RMN à Très hauts Champs \\ UMR 5280 (CNRS / Ecole Normale Supérieure de Lyon / Université Claude Bernard Lyon 1) \\ 5 rue de la Doua, F-69100 Villeurbanne (France)
}

\begin{abstract}
Magic-Angle Spinning (MAS) Nuclear Magnetic Resonance (NMR) is a fastdeveloping technique, capable of complementing solution NMR, X-ray crystallography and electron microscopy for the biophysical characterization of microcrystalline, poorly crystalline or disordered protein samples, such as enzymes, biomolecular assemblies, membraneembedded systems or fibrils. Beyond structures, MAS NMR is an ideal tool for investigation of dynamics, since it is unique in its ability to distinguish static and dynamic disorder, and to characterize not only amplitudes, but also timescales of motion. Building on seminal work on model proteins, the technique is now ripe for widespread application in structural biology. This review briefly summarizes the recent evolutions in biomolecular MAS NMR and accounts for the growing number of systems where this spectroscopy has provided a description of conformational dynamics over the very last few years.
\end{abstract}

Introduction. The experimental determination of protein dynamics at the atomic level is one of the major challenges of modern biology. It requires the characterization of proteins in the 4D space defined by the three space coordinates and a time dimension. Most biophysical techniques are able either to obtain high-resolution structural ensembles (e.g. X- ray diffraction and electron microscopy) or to provide information about their time evolution (e.g. infrared, fluorescence and electron paramagnetic resonance spectroscopies), but not both at the same time. In addition, any biophysical study has to isolate the object of interest from its native environment, which may strongly affect protein behavior. When performing in vitro studies, the experimentalist has to find a balance between the requirements inherent to the technique and the most native conditions for the protein.

Nuclear Magnetic Resonance (NMR) has a particular place among biophysical methods because of its power of revealing protein dynamics with both spatial and temporal resolution. A large arsenal of NMR methods is routinely used to characterize motions of proteins in solution at different timescales. Applications in structural biology include determination of entropic changes upon ligand binding or mutation, characterization of allosteric effects on enzyme function or the indirect observation of folding intermediates. For a large number of systems which are either not soluble or have a prohibitively large molecular weight for solution studies (large complexes, viral capsids, membrane proteins, fibrils, ...), solid-state NMR is a technique of choice. In solid samples, however, anisotropic interactions between spins broaden the NMR lines and add unwanted contributions to the observables which report on dynamics. Magic-Angle Spinning (MAS) of the sample helps improving spectral quality and recovering information about dynamics by averaging these interactions.

The field of MAS NMR has experienced an extremely rapid development over the last two decades, promoted by continuous advances in terms of instrumentation and methodology. High magnetic fields, very fast MAS, cryogenic probes and dynamic nuclear polarization (DNP) speed up today by orders of magnitude the analysis of proteins of considerable size, enabling the investigation of dynamics over a broad range of timescales in crystalline and noncrystalline preparations. This leap forward has fueled the progressive integration of MAS NMR with other mature experimental biophysical techniques and molecular dynamics (MD) simulations, helping in bridging structure to function in a variety of biological processes, such as catalysis, drug binding, viral infectivity, transport across lipid membranes or amyloid fibril formation. 
MAS NMR methods for the measurement of protein dynamics. Molecular dynamics is one of the main information directly accessible from the inspection of MAS NMR correlation spectra as the way itself by which spectra are acquired is sensitive to different motional modes. A first class of experiments are based on the so-called Cross-Polarization (CP) between dipolarcoupled nuclear spins. These sequences tend to select rigid sites as their efficiency scales down with the amplitude of any motion whose frequency is higher than the coupling constant (eg $\sim 10$ and $\sim 20 \mathrm{kHz}$ for ${ }^{1} \mathrm{H}-{ }^{15} \mathrm{~N}$ and ${ }^{1} \mathrm{H}-{ }^{13} \mathrm{C}$ dipolar couplings, respectively). Conversely, regions with very fast mobility are selected by a second class of irradiation schemes which, similarly to solution NMR, make use of weaker isotropic scalar couplings and long coherence evolution periods. Comparing peak intensities in spectra acquired with either of the above techniques is therefore a straightforward way to diagnose whether a protein site belongs to rigid or mobile portions of the molecule [1].

More detail about the motional process can be obtained by measuring site-specifically the anisotropic interaction tensors (Figure 1a). In powdered solids, the observed strengths of these interactions are reduced by motional amplitudes and their asymmetry reports on the geometry of the dynamics. Another approach consists in measuring spin relaxation, which detects the fluctuations of the interaction tensor within an observable-specific "time window" (Figure 1b).

The two above-mentioned techniques have had limited applications in structural biology until recently, as they require long experimental times and site resolution, which is often missing for large molecules also in 2D or 3D correlation spectra. In addition, the presence in solid samples of a strong network of dipolar-coupled protons induces coherent contributions to the spin relaxation observables, impeding their straightforward conversion into dynamics parameters. Two decades of developments in hardware (stronger external magnetic fields, faster MAS, cryogenic probes, Figure $2 \mathrm{a}$ ) and in labelling schemes allowed to remove these hurdles and to enlarge the repertoire of MAS NMR probes of dynamics.

In particular, the progressive extension of the NMR spectra to the ${ }^{1} \mathrm{H}$ dimension by faster MAS rates has revolutionized the field, boosting both resolution and sensitivity, and thus enabling the investigation of more complex protein systems of larger molecular weight, with significantly reduced sample quantities (down to less than $0.5 \mathrm{mg}$ ) [2-4]. New efficient strategies including time-shared experiments [5-7], non-uniform sampling [8], projection spectroscopy [9] and paramagnetic doping $[10,11]$ allow today the accelerated acquisitions of multidimensional spectra for resonance assignment, their rapid automatic evaluation, and their use for the measurement of site-specific dynamics parameters. Most importantly, faster MAS, often in combination with suitable deuteration schemes, reduces or suppresses the unwanted contributions of the ${ }^{1} \mathrm{H}$ network, permitting a quantitative analysis of spin relaxation in terms of conformational dynamics [12].

Hand in hand with this technological leap forward, the quality and quantity of observables for the measurement of protein motions have progressed along two different directions. First, the pool of nuclei used to detect motions has been enriched, now comprising amide ${ }^{15} \mathrm{~N},{ }^{13} \mathrm{CA}$, side-chain ${ }^{13} \mathrm{C}[13,14],{ }^{2} \mathrm{H}[15],{ }^{19} \mathrm{~F}[16,17]$ and the first exploratory reports on ${ }^{1} \mathrm{H}[18,19]$, each nucleus type probing different dynamical modes. Second, new experimental schemes have enabled to widen the observation window towards more diverse motional timescales. Notably, techniques for the quantification of $\mu \mathrm{s}-\mathrm{ms}$ exchange processes (relaxation dispersion methods or Chemical Exchange Saturation Transfer, CEST) have been the focus of many recent developments. This progress in methodology, reviewed in details in $[20,21]$, opens a window to observe relevant phenomena such as protein folding, transport and signaling. The combination of the different experiments available today allows to thoroughly sample dynamics from ps to more than s (Figure 2b).

Other exciting developments on protein dynamics concern MAS NMR experiments over an expanded temperature range, enabled by the availability of new hardware combining sample spinning with cryogenic cooling. These new capabilities allow to isolate motional processes with different activation energies, including methyl rotation, rotamer jumps, hydration dynamics 
$[22,23]$ or the ring flip of aromatic side-chains [14]. For systems undergoing large structural fluctuations, correlation spectra at cryogenic temperatures comprise contributions from the whole (frozen) conformational ensemble. Although this is a further source of inhomogeneous line broadening, and is commonly seen as a bottleneck for applying Dynamic Nuclear Polarization (DNP) to biological samples, line-shape analysis was proposed to probe conformational sampling in frozen preparations of intrinsically disordered proteins or of viral capsid assemblies $[24,25]$. The commercialization of gyrotrons for high magnetic field DNP and cryogenic probe-heads capable of faster MAS (up to $60 \mathrm{kHz}$ ) together with ceaseless innovations in sample formulations, pushes the limits of spectral resolution while keeping high the signal enhancements.

A new perspective for solid-state NMR experiments is the use of controlled freezing process to trap reaction intermediates in processes such as amyloid fibril formation or protein folding and oligomerization. A recent example of 'time-resolved' follow-up of a structural conversion process reached millisecond time resolution thanks to the engineering of a new device for coupling low-temperature MAS NMR with signal enhancements from DNP with rapid mixing and freeze-trapping [26].

In the following, we present an overview of the new insight provided by modern MAS NMR on motions determining protein stability and function (catalytic activity, transport, aggregation propensity) in a variety of samples in crystalline and non-crystalline environments.

Microcrystalline proteins. Crystals and microcrystals have been extensively used as model systems for the development of biomolecular MAS NMR, because the high homogeneity of crystalline preparations guarantees high spectral resolution. Despite the higher order present in these preparations, a growing body of evidence indicates that protein flexibility is preserved in hydrated microcrystals $[27,28]$. Microcrystals stand therefore today on their own ground an ideal platform for the biophysical characterization of protein dynamics and binding by solidstate NMR.

The effect of crystal packing on internal motions has been critically assessed. It was shown that fast ps-ns motions are perfectly conserved, while crystal packing affects amplitudes and timescales of slower collective motions [27-29]. Also in this case, however, NMR parameters are still diagnostic of residual effects, which are reminiscent of the corresponding processes occurring in solution. Most notably, the investigation of dynamics in microcrystals by MAS NMR benefits from the absence of molecular tumbling, which allows to expand the window of motional timescales observable by spin relaxation with respect to the solution counterpart.

As a result, applications of dynamics by MAS NMR in microcrystalline preparations have been shown to be particularly powerful for the elucidation of unforeseen properties of enzymes and to provide a rationale for the unfolding properties of pathologic mutants.

Two intriguing recent examples concern the well-studied human enzymes carbonic anhydrase II (hCAII) and Cu,Zn-superoxide dismutase (SOD). Here, MAS NMR has bridged the gap between X-ray crystallography and solution NMR investigations, revealing unprecedented details on the link between internal dynamics and enzyme activity and stability. Human carbonic anhydrase II (hCAII) is used as a model system for drug design, since a large number of crystallographic structures predict a well-defined structure and a rigid active site. While confirming such rigidity in the ps-ns timescale [30], MAS NMR investigation revealed the presence of slower conformational exchange in the $\mu$ s range in the active site (Figure 3a), a process connected with enzyme activity and abrogated by binding with an inhibitor [31,32]. Exchange over the same timescales was also revealed for the network of water molecules in the active cavity. This suggests that the dynamics of these solvent molecules, which were previously identified as merely structural by diffraction, is tightly linked to catalytic function. SOD is also a very well-known metalloenzyme regulating cellular copper homeostasis and has been largely investigated in the context of its implication in amyloid diseases. When comparing the mature form of $\mathrm{Cu}, \mathrm{Zn}$-SOD with a Cu-depleted intermediate, no structural change was observed. Conversely the investigation of motions in a window from $\mathrm{ps}$ to $\mathrm{ms}$ revealed a 
multimodal response, with the metal acting as a control element and redistributing dynamic processes over multiple timescales. Moreover, a coupling was observed between motions of side chains coordinating the copper in the active site and those of distant backbone elements of the protein which are pivotal for SOD stability and efficient enzymatic activity [33]. The multitimescale dynamics unveiled in these studies by solid-state NMR are particularly difficult to observe using other methods [34], and in the specific case of SOD had escaped previous determinations by solution NMR.

An additional advantage of microcrystalline preparations is the role of the crystal lattice in preserving the native conformation of aggregation-prone proteins. This was particularly relevant in the investigation of conformational dynamics of beta-2 microglobulin and its pathological mutant D76N in microcrystals. The comparative study of the intrinsic dynamics in the two forms revealed in D76N the existence of exchange with a less structured high-energy state, providing a molecular explanation for the reactivity of the pathological mutant at the earliest steps of fibrillization [35]. This represents a new and unexpected spearhead for the investigation of protein folding and misfolding.

Complexes, oligomeric and multimeric assemblies. For large molecules, fast MAS averages anisotropic interactions better than the Brownian motion in solution, and MAS NMR thus provides site-resolved pictures of dynamic processes independently from the molecular size. In addition, crystallinity is not a pre-requisite for obtaining resolved spectra with MAS NMR, making this technique particularly suitable to investigate precipitates of binary proteinprotein or protein-DNA complexes, large modular molecular machines and periodic assemblies such as viral capsids and bacterial virulence factors. In this respect, MAS NMR is uniquely positioned for unveiling allosteric mechanisms, which are of fundamental importance in drug development, and perfectly complements solution NMR studies, where dynamics is typically measured in individual, isolated components of an assembly.

An interesting example is represented by oligomeric enzymatic complexes, where substrates binding to a single subunit modulate function through coordinated conformational transitions. Within an integrated multi-technique approach, MAS NMR allowed to characterize enzyme inhibition and activation in the Caseinolytic protease (ClpP), a tetra-decameric machinery recently emerging as an exciting antibacterial drug target [36]. The study unveiled a concerted allosteric activation of ClpP following binding of a substrate-mimicking inhibitor in the active site, which represents the first proposed rationale for inhibitor-induced activation.

Dynamics plays a crucial role in the morphology and the stability of viral capsids, governing their assembly, disassembly and infectivity. MAS NMR was used to elucidate dynamic allostery in the context of HIV-1 capsids (Figure 3b), where a rigidification of several interfaces essential for higher-order capsid assembly was observed when the capsid binds the host factor protein TRIM5 $\alpha$ [37]. In parallel, MAS NMR, in conjunction with MD simulations, was used to solve the structure of HIV-1 capsid protein tubes, revealing atomic-level detail of a dynamical capsid architecture and providing important guidance for the design of novel inhibitors [38]. For large assemblies formed by high-molecular-weight proteins, segmental isotopic labelling enables MAS NMR experiments that test specific hypotheses concerning a particular, functionally important, set of atoms of interest, obviating the need for complete peak assignment. This approach was used in the context of spherical virus-like particles HIV-1 in order to identify the subtle role of local dynamics at a critical site associated to the binding of an inhibitor of the conversion of immature HIV-1 virions to the mature, infectious state [39].

Sample sedimentation by ultracentrifugation [40] has become an established way to immobilize biomolecular preparations, making them amenable to MAS NMR, while preserving their hydration and their internal dynamics. This represents an ideal approach for providing dynamic pictures of molecular machines and describing the kinetics of the catalysis in relation to the rate of structural changes. For example DnaB helicase, an ATP-fueled catalyst responsible of the unwinding of DNA, was sedimented in complexes with nucleotides and single-stranded DNA [41]. These preparations captured a multitude of intermediate states involved in the 
convoluted reaction pathway of the catalysis. The investigation of conformational changes by MAS NMR highlighted the coupling between the different processes in terms of local variations in dynamics, allowing the authors to propose a mechanism for DNA translocation by DnaB.

Integral membrane proteins. The unique ability of MAS NMR to characterize amplitudes and timescales of motion is particularly interesting for membrane proteins, because their function often involves conformational plasticity, either for transport of small molecules, or for signaling across the membrane. Integral membrane proteins are particularly challenging for biophysical studies due to their particular preparation in lipids or detergents, which induces an inherent inhomogeneity in the NMR sample, and lowers its protein content. In addition, the behavior of these proteins is highly dependent on the membrane-mimicking environment. These difficulties are the driving force and the main targets of the recent developments of MAS NMR. Notably, MAS NMR extends the field of investigation of membrane protein dynamics from studies using detergent solvation to more native environments such as lipid bilayers.

The importance of working in a lipid environment is illustrated by a recent study of the conformational dynamics of human Aquaporin 1 (hAQP1) [42]. A structural model based on site-specific H/D-exchange MAS NMR measurements together with chemical-shifts restrained MD displayed marked differences with the crystallographic structures in detergents over different portions of the molecule. Remarkably, a reduced dynamical behavior was observed in lipids for an extracellular loop, critical for water permeation, suggesting a role of dynamics on the regulation of water transport in hAQP1 [43].

Another crucial feature of membrane proteins, tightly linked to the motional behavior of the molecule and to their function, is the protonation state of the chemical groups, directly accessible from the ${ }^{1} \mathrm{H}$ chemical shifts. This aspect has been largely investigated in the Influenza M2 protein, as the protonation/deprotonation equilibria of side chains exposed in the channel are required for proton conduction [44-46]. In particular, backbone and side-chain dynamic processes were shown to couple with these processes on timescales from ps to $\mu s$ [47]. Similarly, the protonation dynamics of the active site of bacteriorhodopsin was monitored by ${ }^{1} \mathrm{H}$ chemical shift exchange experiments and revealed slow oscillatory motions of protons in the dark state. Such protonation dynamics is thought to be disrupted in the light-activated states, thus promoting the functional proton pumping [48] .

The advantages associated to ${ }^{1} \mathrm{H}$ detection and fast MAS in spin relaxation studies of membrane proteins have been first applied to the precise determination of collective wobbling motions of individual helices in the seven-helical transmembrane protein, Anabaena Sensory Rhodopsin (ASR) [49] and of the beta-barrel of the Outer Membrane Protein A (OmpA) [50].

Following these studies, ${ }^{1} \mathrm{H}$-detected spin relaxation techniques have provided a deeper understanding of gating mechanisms in different classes of transporters. A first example has been the characterization of microsecond-exchange mechanisms in the transmembrane helix 5 of the protease GlpG. Here ${ }^{15} \mathrm{~N} \mathrm{R}_{1 p}$ relaxation dispersion experiments enriched the picture of the interplay between open and closed forms in Rhomboid proteases [51].

A second notable example has been the complete ${ }^{1} \mathrm{H}$-detected MAS NMR structure determination of the alkane transporter AlkL in lipid bilayers. Combined to spin relaxation measurements and MD simulations [52], this has solved a controversy on the mechanism of permeation of the outer membrane of bacteria by hydrophobic molecules. The elucidation of the conformational dynamics of the extracellular loops on different timescales has allowed to understand the subtle balance between the rigidity necessary to confine substrates at the pore entrance and the plasticity required for lateral exit towards the hydrophobic portion of the outer membrane (Figure 3c).

The sensitivity boost due to ${ }^{1} \mathrm{H}$-detection has been also brightly exploited to investigate the modal gating switches of the $\mathrm{K}+$ channel $\mathrm{KcsA}$ by rapidly scanning chemical shifts and relaxation properties of the wild type protein and of three mutants mimicking different naturally occurring gating modes [53]. The wealth of information accessible by MAS NMR allowed the 
authors to highlight the molecular determinants and the pivotal role of conformational dynamics of the selectivity filter in the gating mechanism.

The recent development of ${ }^{19} \mathrm{~F}$ MAS NMR has provided an alternative approach for the investigation of structural dynamics in membrane proteins. The large gyromagnetic ratio and chemical-shift range make fluorine useful not only as a "nanometric ruler" to measure long interatomic distances through homonuclear and heteronuclear dipolar couplings [17,54], but also as a sensitive probe of local conformational changes. This was exploited for the study of the bacterial transporter EmrE, primarily involved in the drug-efflux mechanism developed by bacteria as an antibiotic resistance. $\mathrm{A}^{19} \mathrm{~F}$-labeled substrate enabled to both solve the structure of the binding pocket and elucidate the dynamics of the adduct [55]. In particular, ${ }^{19} \mathrm{~F}$ exchange spectroscopy revealed the existence of ms conformational fluctuations in the spacious binding pocket, justifying the multidrug specificity of EmrE.

All above mentioned studies are carried out on proteins that undergo prior isolation and reconstitution in lipid bilayers mimicking their natural environment. An exciting possibility, opened by MAS NMR, is to investigate proteins within native membranes [56-58], where the lipid composition and orientation but also the presence of other proteins strongly affects their behavior [57,59]. An example of the impact of these factors on protein dynamics is the investigation of the Light-harvesting complex II (LHCII) in native thylakoid membrane [60]. The comparison of dynamics-edited correlation spectra of both LHCII reconstituted in lipid bilayers and in thylakoid membranes highlights key protein regions for which conformational equilibria are shifted by the lipidic environment.

In this context, DNP is playing a growing role in the study of membrane proteins as the sensitivity gains provided by this methods allow to detect protein signals in more and more dilute environments. Pioneering studies already demonstrated the potential of this technique for the investigation of conformational ensembles of large protein complexes [61] and of metastable functional states [62].

Fibrils. MAS NMR proved to be a powerful technique for the study of architecture of amyloid fibrils, a necessary step for understanding the mechanisms underlying aggregation. Although fibrils are commonly seen as highly rigid structures, multiple MAS NMR investigations have described the presence of extensive motions in these assemblies. Particularly striking is the example of the huntingtin exon-1 (HTTex1) aggregates where the residues of the disordered C-terminus part, which may have a role in HTTex1 toxicity, reorient completely in the ps-ns timescale [63].

Understanding the fine distribution of dynamics and water accessibility is an important data to understand fibrils assembly and stability. This was shown on tau fibrils prepared in vitro, in which water-edited spectra revealed that only a small water pool is present in the core of the $\beta$-sheets hairpin and that extended hydration in the repeat is prevented by other protein segments. Also, high order parameters revealed a general rigidity of the backbone in the external segment, suggesting cross- $\beta$ hydrogen bonding involved in the 3D folding [64]. The combination of this observation with spectral editing could reveal a heterogeneous dynamic behavior among different molecules in the sample, as a fraction of the protein subunits are actually disordered in this region [65].

Interestingly, by applying relaxation dispersion methods, Shannon et al. were able to describe conformations in exchange at a rate of $10^{3}-10^{4} \mathrm{~Hz}$ in water-exposed segments of the Y145Stop human prion protein fibrils [66]. Such slow motions were also observed in the fungal prion HET-s(218-289) amyloids [67] (Figure 3d). In the latter study, the thorough description of protein dynamics by MAS NMR at different timescales validates MD simulations, which in turn enabled to assign the observed slow dynamics to backbone motions correlated over numerous protein layers.

By freeze-trapping aggregation intermediates, multiple aspects of dynamical structure conversions can be followed with millisecond time resolution, including changes in secondary structure at specific isotopically labeled sites, formation of intramolecular and intermolecular 
contacts between specific pairs of labeled residues, and variation of overall structural order. In the peptide melittin, $\mathrm{pH}$-induced helical folding and self-assembly were shown to occur simultaneously in less than $10 \mathrm{~ms}$, while a final tetrameric state developed from the conversion of conformationally ordered antiparallel melittin dimers on a much slower timescale of tens of ms [26]. This approach opens a promising avenue for the investigation of the mechanism of fibril formation and protein-protein recognition.

Outlook. The examples reviewed above, which represent by no means an exhaustive list, give the flavor of what MAS NMR can bring to field of structural biology. This list is destined to expand through a deeper integration with other biophysical and computational techniques, a stronger interface with cell biologists and virologists, and the development of improved protocols for the preparation of targets not compatible with bacterial cultures.

Given the evident role played by hardware evolution in the development of the technique, we anticipate a major step forward in the field with the implementation of the next generation of 1.2 GHz magnets, of probes capable of spinning at faster rates [68], and of gyrotrons (and polarizing agents) adapted to higher magnetic fields $[69,70]$. Coupled to improved protocols for in cell investigations [58,71], this will allow to push even farther the current resolution and sensitivity barriers and enable the detailed investigation of protein dynamics in native conditions.

\section{Acknowledgements}

Financial support from the CNRS (IR-RMN FR3050) and from the European Research Council (ERC) under the European Union's Horizon 2020 research and innovation programme (ERC2015-CoG GA n648974) are gratefully acknowledged.

\section{Figure captions}

Figure 1. Schematic representation of two classes of MAS NMR experiments sensitive to biomolecular motions: (a) direct measurement of scaled (motion-averaged) anisotropic interaction tensors (here, dipolar couplings), (b) spin relaxation caused by fluctuations of the anisotropic interactions in observable-specific time windows. Blue indicates observables related to rigid fragments, while red represents those associated to dynamical regions. Site specificity is ensured by associating the above measurements to the different MAS NMR signals resolved in multidimensional correlation spectra.

Figure 2. Left: Milestones in development of MAS NMR instrumentation over the last twenty years, enabling the investigation of site-specific protein dynamics at room and cryogenic temperatures. Right: Sensitivity of MAS NMR experiments to different timescales of conformational dynamics, from $\mathrm{ps}$ to $\mathrm{s}$. The same color gradient used on the left panel indicates here the approximate year of introduction in the community.

Figure 3. Representation of conformational dynamics as recently established by MAS NMR in four classes of samples: microcrystalline proteins (a), viral capsid assemblies (b), membrane proteins (c), fibrils (d). Proteins are represented as cylinders, whose thickness and color (grey to red) highlight areas undergoing conformational dynamics, and specifically: (a) $\mu \mathrm{s}$ conformational exchange in the active site loop of hCAll, assessed by ${ }^{15} \mathrm{~N}$ relaxation dispersion experiments [34]; (b) flexibility of the CypA interaction loop in tubular assemblies of the HIV-1 capsid protein, by ${ }^{1} \mathrm{H}-{ }^{15} \mathrm{~N}$ dipolar couplings measurements [72]; (c) ns- $\mu$ s backbone motions in AlkL involved in short-chain alkane transport, by ${ }^{15} \mathrm{~N} \mathrm{R}_{1 p}$ spin relaxation [52]; (d) concerted nsus motions over multiple layers of HET-s(218-289) fibrils, by optimized-detectors analysis of ${ }^{15} \mathrm{~N}$ relaxation rates [67]. In all panels, pictures of sample preparations are shown in the insets. 


\section{Bibliography}

1. Matlahov I, Wel PCA Van Der: Hidden motions and motion-induced invisibility: dynamics-based spectral editing in solid-state NMR. Methods 2018, doi:10.1016/j.ymeth.2018.04.015.

2. Agarwal V, Penzel S, Szekely K, Cadalbert R, Testori E, Oss A, Past J, Samoson A, Ernst M, Böckmann A, et al.: De Novo 3D Structure Determination from Submilligram Protein Samples by Solid-State $100 \mathrm{kHz}$ MAS NMR Spectroscopy. Angew Chemie Int Ed 2014, 53:12253-12256.

3. Andreas LB, Jaudzems K, Stanek J, Lalli D, Bertarello A, Le Marchand T, Cala-De Paepe D, Kotelovica S, Akopjana I, Knott B, et al.: Structure of fully protonated proteins by proton-detected magic-angle spinning NMR. Proc Natl Acad Sci USA 2016, 113:9187-9192.

4. Vasa SK, Rovó P, Linser R: Protons as Versatile Reporters in Solid-State NMR Spectroscopy. Acc Chem Res 2018, 51:1386-1395.

5. Stanek J, Schubeis T, Paluch P, Güntert P, Andreas LB, Pintacuda G: Automated Backbone NMR Resonance Assignment of Large Proteins Using Redundant Linking from a Single Simultaneous Acquisition. J Am Chem Soc 2020, 142:57935799.

6. Sharma K, Vipin PKM, Kaustubh A: Simultaneous recording of intra - and inter residue linking experiments for backbone assignments in proteins at MAS frequencies higher than $60 \mathrm{kHz}$. J Biomol NMR 2020, doi:10.1007/s10858-01900292-y.

7. Gallo A, Franks WT, Lewandowski JR: A suite of solid-state NMR experiments to utilize orphaned magnetization for assignment of proteins using parallel high and low gamma detection. J Magn Reson 2019, 305:219-231.

8. Polenova T: A Time-Saving Strategy for MAS NMR Spectroscopy by Combining Nonuniform Sampling and Paramagnetic Relaxation Assisted Condensed Data Collection. 2012, doi:10.1021/jp3005794.

9. Orton HW, Stanek J, Schubeis T, Foucaudeau D, Ollier C, Draney AW, Le Marchand T, Cala-De Paepe D, Felli IC, Pierattelli R, et al.: Protein NMR Resonance Assignment without Spectral Analysis: 5D SOlid-State Automated Projection SpectroscopY (SO-APSY). Angew Chemie Int Ed 2020, 59:2380-2384.

10. Wickramasinghe NP, Parthasarathy S, Jones CR, Bhardwaj C, Long F, Kotecha M, Mehboob S, Fung LW, Past J, Samoson A, et al.: Nanomole-scale protein solidstate NMR by breaking intrinsic 1 H T boundaries. 2009, 6.

11. Öster C, Kosol S, Lewandowski JR: Quantifying Microsecond Exchange in Large Protein Complexes with Accelerated Relaxation Dispersion Experiments in the Solid State. Sci Rep 2019, 9:11082.

12. Schanda $P$, Ernst $M$ : Studying dynamics by magic-angle spinning solid-state NMR spectroscopy: Principles and applications to biomolecules. Prog Nucl Magn Reson Spectrosc 2016, 96:1-46.

13. Xue K, Mamone S, Koch B, Sarkar R, Reif B: Determination of methyl order parameters using solid state NMR under off magic angle spinning. $J$ Biomol NMR 2019, 73:471-475.

*14. Gauto DF, Macek P, Barducci A, Fraga H, Hessel A, Terauchi T, Gajan D, Miyanoiri Y, Boisbouvier J, Lichtenecker R, et al.: Aromatic Ring Dynamics, Thermal Activation, and Transient Conformations of a 468 kDa Enzyme by Specific 1H-13C Labeling 
and Fast Magic-Angle Spinning NMR. J Am Chem Soc 2019, 141:11183-11195.

This study probes the dynamics of Phe side chains in the 0.5 MDa TET2 enzyme assembly using room and cryogenic temperature MAS NMR and uses the first ${ }^{13} C R_{10}$ MAS NMR relaxation-dispersion measurements to detect $\mu$ s conformational changes at the entry and exit pores of the catalytic chamber.

15. Shi X, Rienstra CM: Site-Specific Internal Motions in GB1 Protein Microcrystals Revealed by 3D2H-13C-13C Solid-State NMR Spectroscopy. J Am Chem Soc 2016, 138:4105-4119.

16. Wang M, Lu M, Fritz MP, Quinn CM, Byeon IJL, Byeon CH, Struppe J, Maas W, Gronenborn AM, Polenova T: Fast Magic-Angle Spinning 19F NMR Spectroscopy of HIV-1 Capsid Protein Assemblies. Angew Chemie Int Ed 2018, 57:16375-16379.

17. Roos M, Wang T, Shcherbakov AA, Hong M: Fast Magic-Angle-Spinning 19F Spin Exchange NMR for Determining Nanometer 19F-19F Distances in Proteins and Pharmaceutical Compounds. J Phys Chem B 2018, doi:10.1021/acs.jpcb.8b00310.

18. Struppe J, Quinn CM, Lu M, Wang M, Hou G, Lu X, Kraus J, Andreas LB, Stanek J, Lalli $\mathrm{D}$, et al.: Expanding the horizons for structural analysis of fully protonated protein assemblies by NMR spectroscopy at MAS frequencies above $100 \mathrm{kHz}$. Solid State Nucl Magn Reson 2017, 87:117-125.

19. Rovó P, Smith CA, Gauto D, De Groot BL, Schanda P, Linser R: Mechanistic Insights into Microsecond Time-Scale Motion of Solid Proteins Using Complementary $15 \mathrm{~N}$ and $1 \mathrm{H}$ Relaxation Dispersion Techniques. J Am Chem Soc 2019, 141:858-869.

*20. Schanda P: Relaxing with liquids and solids - A perspective on biomolecular dynamics. J Magn Reson 2019, 306:180-186.

This review presents recent advances and open challenges in the measurement of spin relaxation by solution and MAS NMR.

*21. Rovó P: Recent advances in solid-state relaxation dispersion techniques. Solid State Nucl Magn Reson 2020, 108:101665.

This review presents a comprehensive theoretical and experimental framework for the acquisition and the interpretation of $R_{1 \rho}$-based relaxation dispersion techniques in rotating solids.

22. Lewandowski JR, Halse ME, Blackledge M, Emsley L: Direct observation of hierarchical protein dynamics. Science 2015, 348:578-581.

23. Busi B, Yarava JR, Hofstetter A, Salvi N, Cala-De Paepe D, Lewandowski JR, Blackledge M, Emsley L: Probing Protein Dynamics Using Multifield Variable Temperature NMR Relaxation and Molecular Dynamics Simulation. J Phys Chem B 2018, 122:9697-9702.

24. Uluca B, Viennet T, Petrović D, Shaykhalishahi H, Weirich F, Gönülalan A, Strodel B, Etzkorn M, Hoyer W, Heise H: DNP-Enhanced MAS NMR: A Tool to Snapshot Conformational Ensembles of a-Synuclein in Different States. Biophys J 2018, 114:1614-1623.

25. Gupta R, Zhang H, Lu M, Hou G, Caporini M, Rosay M, Maas W, Struppe J, Ahn J, Byeon IJL, et al.: Dynamic Nuclear Polarization Magic-Angle Spinning Nuclear Magnetic Resonance Combined with Molecular Dynamics Simulations Permits Detection of Order and Disorder in Viral Assemblies. J Phys Chem B 2019, 123:5048-5058.

**26. Jeon J, Thurber KR, Ghirlando R, Yau WM, Tycko R: Application of millisecond time-resolved solid state NMR to the kinetics and mechanism of melittin self- 
assembly. Proc Natl Acad Sci USA 2019, 116:16717-16722.

This paper presents an innovative apparatus which combines rapid mixing, freezetrapping and DNP signal enhancement to follow the structural conversion of reaction intermediates with ms resolution.

27. Agarwal V, Xue Y, Reif B, Skrynnikov NR: Protein Side-Chain Dynamics As Observed by Solution- and Solid-State NMR Spectroscopy: A Similarity Revealed. J Am Chem Soc 2008, 130:16611-16621.

28. Mollica L, Baias M, Lewandowski JR, Wylie BJ, Sperling LJ, Rienstra CM, Emsley L, Blackledge M: Atomic-resolution structural dynamics in crystalline proteins from NMR and molecular simulation. J Phys Chem Lett 2012, 3:3657-3662.

29. Kurauskas V, Izmailov SA, Rogacheva ON, Hessel A, Ayala I, Woodhouse J, Shilova A, Xue Y, Yuwen T, Coquelle N, et al.: Slow conformational exchange and overall rocking motion in ubiquitin protein crystals. Nat Commun 2017, 8:1-33.

30. Vasa SK, Singh H, Rovó P, Linser R: Dynamics and Interactions of a 29-kDa Human Enzyme Studied by Solid-State NMR. J Phys Chem Lett 2018, doi:10.1021/acs.jpclett.8b00110.

**31. Singh H, Vasa SK, Jangra H, Rovó P, Päslack C, Das CK, Zipse H, Schäfer L V., Linser R: Fast Microsecond Dynamics of the Protein-Water Network in the Active Site of Human Carbonic Anhydrase II Studied by Solid-State NMR Spectroscopy. J Am Chem Soc 2019, 141:19276-19288.

Relaxation dispersion techniques are employed to connect the enzymatic activity of $h C A l l$ with the $\mu s$-dynamics of the water pocket architecture at the active site.

32. Vasa SK, Singh H, Grohe K, Linser R: Assessment of a Large Enzyme-Drug Complex by Proton-Detected Solid-State NMR Spectroscopy without Deuteration. Angew Chemie Int Ed 2019, 58:5758-5762.

33. Bonaccorsi M, Knight MJ, Le Marchand T, Dannatt HRW, Schubeis T, Salmon L, Felli IC, Emsley L, Pierattelli R, Pintacuda G: Multimodal Response to Copper Binding in Superoxide Dismutase Dynamics. J Am Chem Soc 2020, 142:19660-19667.

34. Singh H, Das CK, Vasa SK, Grohe K, Schäfer L V., Linser R: The Active Site of a Prototypical "Rigid" Drug Target is Marked by Extensive Conformational Dynamics. Angew Chemie Int Ed 2020, 59:22916-22921.

35. Le Marchand T, De Rosa M, Salvi N, Sala BM, Andreas LB, Barbet-Massin E, Sormanni P, Barbiroli A, Porcari R, Sousa Mota C, et al.: Conformational dynamics in crystals reveal the molecular bases for D76N beta-2 microglobulin aggregation propensity. Nat Commun 2018, 9:1-11.

36. Felix J, Weinhäupl K, Chipot C, Dehez F, Hessel A, Gauto DF, Morlot C, Abian O, Gutsche I, Velazquez-Campoy A, et al.: Mechanism of the allosteric activation of the ClpP protease machinery by substrates and active-site inhibitors. Sci Adv 2019, 5:eaaw3818.

**37. Quinn CM, Wang M, Fritz MP, Runge B, Ahn J, Xu C, Perilla JR, Gronenborn AM, Polenova T: Dynamic regulation of HIV-1 capsid interaction with the restriction factor TRIM5 $\alpha$ identified by magic-angle spinning NMR and molecular dynamics simulations. Proc Natl Acad Sci USA 2018, 115:11519-11524.

MAS NMR techniques and MD simulations are combined to describe the structural dynamics of pentameric and hexameric HIV-1 capsid assemblies and to elucidate their dynamic allosteric interaction with the restriction factor TRIM5 $\alpha$.

38. Lu M, Russell RW, Bryer AJ, Quinn CM, Hou G, Zhang H, Schwieters CD, Perilla JR, Gronenborn AM, Polenova T: Atomic-resolution structure of HIV-1 capsid tubes 
by magic-angle spinning NMR. Nat Struct Mol Biol 2020, 27:863-869.

39. Gupta S, Tycko R: Segmental isotopic labeling of HIV-1 capsid protein assemblies for solid state NMR. J Biomol NMR 2018, 70:103-114.

40. Bertini I, Luchinat C, Parigi G, Ravera E, Reif B, Turano P: Solid-state NMR of proteins sedimented by ultracentrifugation. Proc Natl Acad Sci USA 2011, 108:10396-10399.

41. Wiegand T, Cadalbert R, Lacabanne D, Timmins J, Terradot L, Böckmann A, Meier $\mathrm{BH}$ : The conformational changes coupling ATP hydrolysis and translocation in a bacterial DnaB helicase. Nat Commun 2019, 10:1-11.

42. Wang $S$, Ing $C$, Emami S, Jiang $Y$, Liang $H$, Pomès R, Brown LS, Ladizhansky V: Structure and Dynamics of Extracellular Loops in Human Aquaporin-1 from Solid-State NMR and Molecular Dynamics. J Phys Chem B 2016, 120:9887-9902.

43. Dingwell DA, Brown LS, Ladizhansky V: Structure of the Functionally Important Extracellular Loop C of Human Aquaporin 1 Obtained by Solid-State NMR under Nearly Physiological Conditions. J Phys Chem B 2019, 123:7700-7710.

44. Kwon B, Roos M, Mandala VS, Shcherbakov AA, Hong M: Elucidating Relayed Proton Transfer through a His-Trp-His Triad of a Transmembrane Proton Channel by Solid-State NMR. J Mol Biol 2019, 431:2554-2566.

45. Movellan KT, Wegstroth M, Overkamp K, Leonov A, Becker S, Andreas LB: Imidazole - Imidazole Hydrogen Bonding in the pH-Sensing Histidine Side Chains of In $\mathrm{fl}$ uenza A M2. 2020, doi:10.1021/jacs.9b10984.

46. Fu R, Miao Y, Qin H, Cross TA: Observation of the Imidazole-Imidazolium Hydrogen Bonds Responsible for Selective Proton Conductance in the In fl uenza A M2 Channel. 2020, doi:10.1021/jacs.9b09985.

47. Mandala VS, Gelenter MD, Hong M: Transport-Relevant Protein Conformational Dynamics and Water Dynamics on Multiple Time Scales in an Archetypal Proton Channel: Insights from Solid-State NMR. J Am Chem Soc 2018, 140:1514-1524.

48. Friedrich D, Brünig FN, Nieuwkoop AJ, Netz RR, Hegemann $P$, Oschkinat $H$ : Collective exchange processes reveal an active site proton cage in bacteriorhodopsin. Commun Biol 2020, 3:4.

49. Good D, Pham C, Jagas J, Lewandowski JR, Ladizhansky V: Solid-State NMR Provides Evidence for Small-Amplitude Slow Domain Motions in a Multispanning Transmembrane a-Helical Protein. J Am Chem Soc 2017, 139:9246-9258.

50. Saurel O, lordanov I, Nars G, Demange P, Le Marchand T, Andreas LB, Pintacuda G, Milon A: Local and Global Dynamics in Klebsiella pneumoniae Outer Membrane Protein a in Lipid Bilayers Probed at Atomic Resolution. J Am Chem Soc 2017, 139:1590-1597.

51. Shi C, Öster C, Bohg C, Li L, Lange S, Chevelkov V, Lange A: Structure and Dynamics of the Rhomboid Protease GIpG in Liposomes Studied by Solid-State NMR. J Am Chem Soc 2019, 141:17314-17321.

**52. Schubeis T, Le Marchand T, Daday C, Kopec W, Movellan KT, Stanek J, Schwarzer TS, Castiglione K, de Groot BL, Pintacuda G, et al.: A $\beta$-barrel for oil transport through lipid membranes: Dynamic NMR structures of AlkL. Proc Natl Acad Sci USA 2020, 117:21014-21021.

This paper represents the first atomic-level structure determination of a membrane protein in lipid bilayers by ${ }^{1} \mathrm{H}$-detected NMR. The methodology is expanded to detect structural dynamics, demonstrating the functional cycle of the protein and the permeation 
of hydrophobic substrates into the membrane.

*53. Jekhmane S, Medeiros-Silva J, Li J, Kümmerer F, Müller-Hermes C, Baldus M, Roux $B$, Weingarth M: Shifts in the selectivity filter dynamics cause modal gating in $\mathbf{K}+$ channels. Nat Commun 2019, 10:123.

The paper provides a high-resolution perspective on the conformational dynamics at the origin of the random shifts between the gating modes of the of $\mathrm{K}^{+}$channel $\mathrm{KcsA}$, explaining a widespread regulatory mechanism in ion channels.

54. Shcherbakov AA, Mandala VS, Hong M: High-Sensitivity Detection of Nanometer 1H-19F Distances for Protein Structure Determination by 1H-Detected Fast MAS NMR. J Phys Chem B 2019, 123:4387-4391.

**55. Shcherbakov AA, Hisao G, Mandala VS, Thomas NE, Soltani M, Salter EA, Davis JH, Henzler-Wildman KA, Hong M: Structure and dynamics of the drug-bound bacterial transporter EmrE in lipid bilayers. Nat Commun 2021, 12:172.

${ }^{19} \mathrm{~F}$ MAS NMR is exploited to determine the structure of the substrate-bound form of a bacterial transporter in lipid bilayers and to assess the dynamics of the substrate in the binding pocket.

56. Pinto $C$, Mance $D$, Julien $M$, Daniels $M$, Weingarth $M$, Baldus $M$ : Studying assembly of the BAM complex in native membranes by cellular solid-state NMR spectroscopy. J Struct Biol 2019, 206:1-11.

57. Singh C, Lee H, Tian Y, Schesser Bartra S, Hower S, Fujimoto LM, Yao Y, Ivanov SA, Shaikhutdinova RZ, Anisimov AP, et al.: Mutually constructive roles of Ail and LPS in Yersinia pestis serum survival . Mol Microbiol 2020, doi:10.1111/mmi.14530.

58. Narasimhan S, Pinto C, Lucini Paioni A, van der Zwan J, Folkers GE, Baldus M: Characterizing proteins in a native bacterial environment using solid-state NMR spectroscopy. Nat Protoc 2021, doi:10.1038/s41596-020-00439-4.

59. Chipot C, Dehez F, Schnell JR, Zitzmann N, Pebay-Peyroula E, Catoire LJ, Miroux B, Kunji ERS, Veglia G, Cross TA, et al.: Perturbations of Native Membrane Protein Structure in Alkyl Phosphocholine Detergents: A Critical Assessment of NMR and Biophysical Studies. Chem Rev 2018, 118:3559-3607.

60. Azadi-Chegeni F, Ward ME, Perin G, Simionato D, Morosinotto T, Baldus M, Pandit A: Conformational Dynamics of Light-Harvesting Complex II in a Native Membrane Environment. Biophys J 2020, doi:10.1016/j.bpj.2020.11.2265.

61. Spadaccini R, Kaur H, Becker-Baldus J, Glaubitz C: The effect of drug binding on specific sites in transmembrane helices 4 and 6 of the ABC exporter MsbA studied by DNP-enhanced solid-state NMR. Biochim Biophys Acta - Biomembr 2018, 1860:833-840.

62. Maciejko J, Kaur J, Becker-Baldus J, Glaubitz C: Photocycle-dependent conformational changes in the proteorhodopsin cross-protomer Asp-His-Trp triad revealed by DNP-enhanced MAS-NMR. Proc Natl Acad Sci USA 2019, 116:8342-8349.

63. Caulkins BG, Cervantes SA, Isas JM, Siemer AB: Dynamics of the Proline-Rich CTerminus of Huntingtin Exon-1 Fibrils. J Phys Chem B 2018, 122:9507-9515.

64. Dregni AJ, Duan P, Hong M: Hydration and Dynamics of Full-Length Tau Amyloid Fibrils Investigated by Solid-State Nuclear Magnetic Resonance. Biochemistry 2020, 59:2237-2248.

65. Dregni AJ, Mandala VS, Wu H, Elkins MR, Wang HK, Hung I, DeGrado WF, Hong M: In vitro $0 N 4 R$ tau fibrils contain a monomorphic $\beta$-sheet core enclosed by dynamically heterogeneous fuzzy coat segments. Proc Natl Acad Sci USA 2019, 
116:16357-16366.

66. Shannon MD, Theint T, Mukhopadhyay D, Surewicz K, Surewicz WK, Marion D, Schanda P, Jaroniec CP: Conformational Dynamics in the Core of Human Y145Stop Prion Protein Amyloid Probed by Relaxation Dispersion NMR. ChemPhysChem 2019, 20:311-317.

67. Smith AA, Ernst M, Riniker S, Meier BH: Localized and Collective Motions in HET$\mathbf{s}(218-289)$ Fibrils from Combined NMR Relaxation and MD Simulation. Angew Chemie Int Ed 2019, 58:9383-9388.

68. Samoson A: H-Mas. J Magn Reson 2019, 306:167-172.

69. Lund A, Casano G, Menzildjian G, Kaushik M, Stevanato G, Yulikov M, Jabbour R, Wisser D, Renom-Carrasco M, Thieuleux C, et al.: TinyPols: a family of watersoluble binitroxides tailored for dynamic nuclear polarization enhanced NMR spectroscopy at 18.8 and 21.1 T. Chem Sci 2020, 11:2810-2818.

70. Berruyer P, Björgvinsdóttir S, Bertarello A, Stevanato G, Rao Y, Karthikeyan G, Casano G, Ouari O, Lelli M, Reiter C, et al.: Dynamic Nuclear Polarization Enhancement of 200 at 21.15 T Enabled by $65 \mathrm{kHz}$ Magic Angle Spinning. J Phys Chem Lett 2020, 11:8386-8391.

71. Frederick KK, Michaelis VK, Corzilius B, Ong TC, Jacavone AC, Griffin RG, Lindquist S: Sensitivity-Enhanced NMR Reveals Alterations in Protein Structure by Cellular Milieus. Cell 2015, 163:620-628.

72. Lu M, Hou G, Zhang H, Suiter CL, Ahn J, Byeon IJL, Perilla JR, Langmead CJ, Hung I, Gor'kov PL, et al.: Dynamic allostery governs cyclophilin A-HIV capsid interplay. Proc Natl Acad Sci USA 2015, 112:14617-14622. 

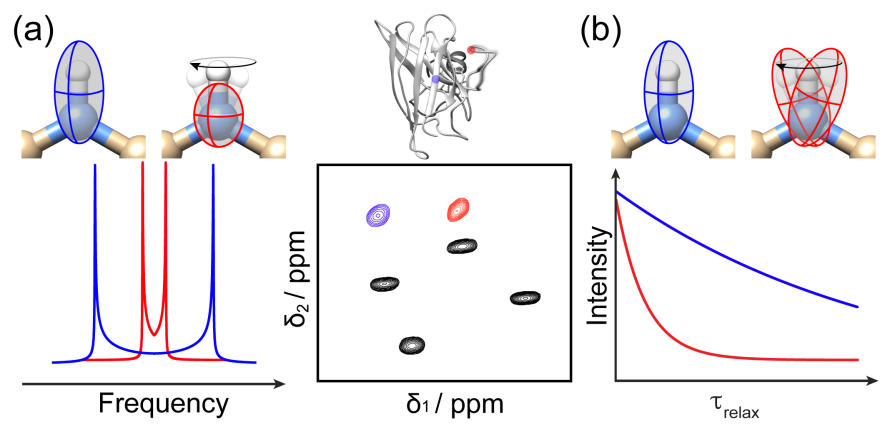

Figure 1.

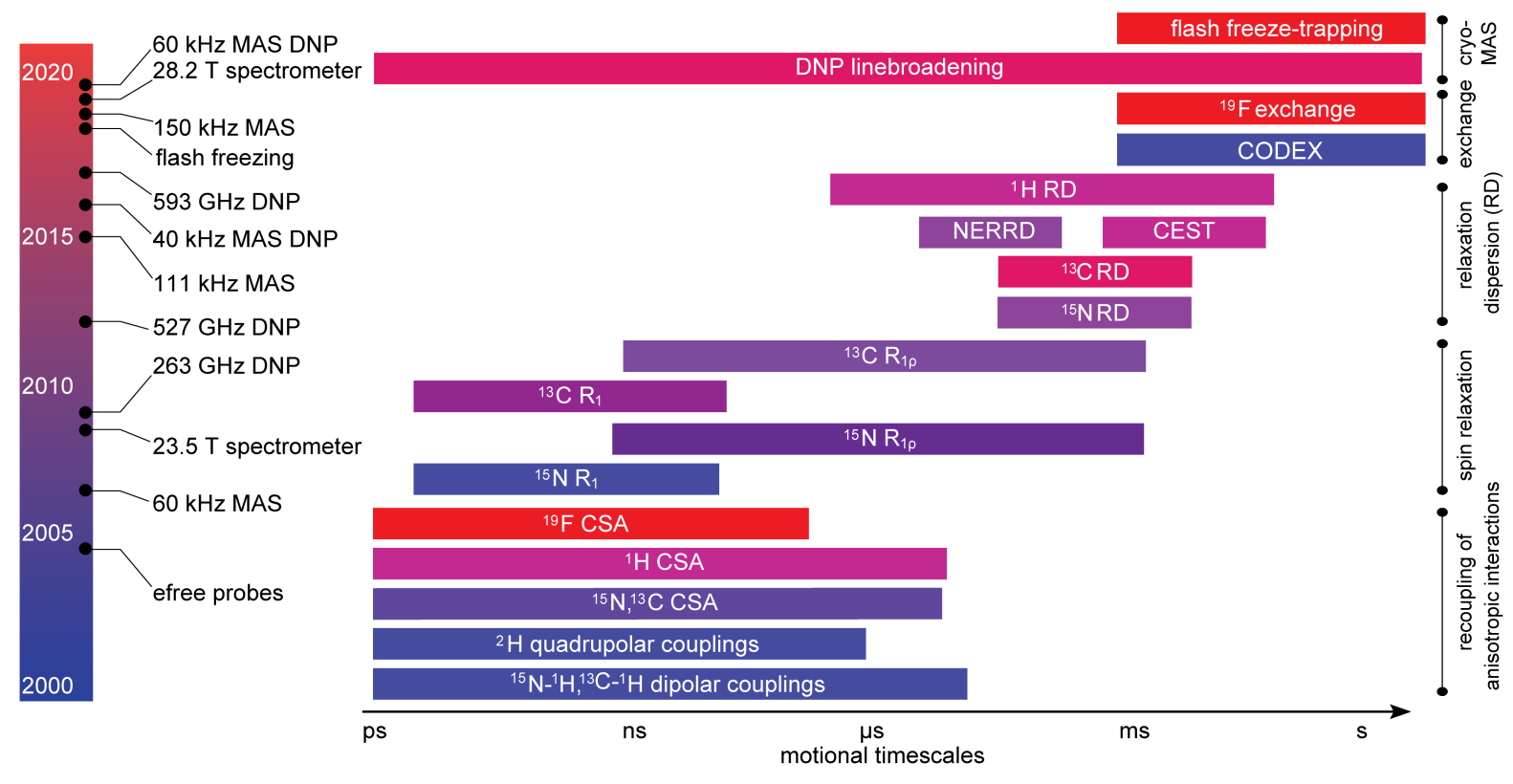

Figure 2.
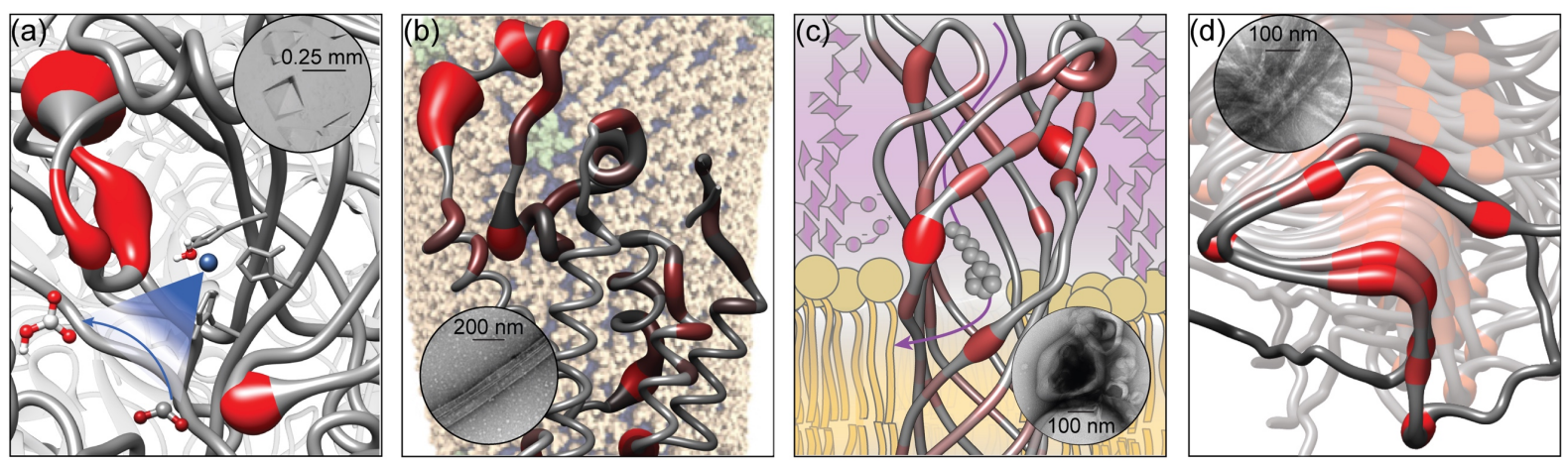

Figure 3. 